\title{
Virtual Transformation and the Use of Social Media: Cardiac Electrophysiology Education in the Post-COVID-19 Era
}

Janet K. Han, $M D^{1, *}{ }^{*}$

Tina Baykaner, $M D^{2}$

Christopher V. DeSimone, MD, $P h D^{3}$

Susan P. Etheridge, $M D^{4}$

Piotr Futyma, $M D^{5}$

Sandeep A. Saha, $M D^{6}$

Martin K. Stiles, MB ChB, PhD ${ }^{7}$

Rakesh Gopinathannair, $M D^{8}$

Rajesh Kabra, $M D^{9}$

Faisal M. Merchant, MD ${ }^{10}$

\section{Address}

*,1Division of Cardiology, David Geffen School of Medicine at the University of California Los Angeles and VA Greater Los Angeles Healthcare System, 11301 Wilshire Blvd 111E, Los Angeles, CA 90073, USA

Email: janethan@mednet.ucla.edu

${ }^{2}$ Division of Cardiovascular Medicine, Stanford University, Palo Alto, CA, USA

${ }^{3}$ Department of Cardiovascular Medicine, Mayo Clinic College of Medicine, Rochester, MN, USA

${ }^{4}$ Division of Pediatric Cardiology, University of Utah and Primary Children's Hospital, Salt Lake City, UT, USA

${ }^{5}$ Medical College of Rzeszow University and St. Joseph's Heart Rhythm Center, Rzeszów, Poland

${ }^{6}$ Oregon Heart Center, Salem, OR, USA

${ }^{7}$ Waikato Clinical School Faculty of Medicine and Health Science, The University of Auckland, Hamilton, New Zealand

${ }^{8}$ Kansas City Heart Rhythm Institute \& Research Foundation, Overland Park, KS, USA

${ }^{9}$ Division of Cardiology, University of Tennessee Health Science Center, Memphis, TN, USA

${ }^{10}$ Division of Cardiovascular Disease, Section of Cardiac Electrophysiology and Pacing, Emory University School of Medicine, Atlanta, GA, USA 
This is a U.S. government work and not under copyright protection in the U.S.; foreign copyright protection may apply 2021

This article is part of the Topical Collection on Arrhythmia

Keywords Cardiac electrophysiology - Digital transformation - Fellowship education - Virtual education . Social media

\begin{abstract}
Purpose of Review The COVID-19 pandemic has significantly impacted the delivery of education for all specialties, including cardiac electrophysiology. This review will provide an overview of the COVID-19 spurred digital transformation of electrophysiology education for practicing clinicians and trainees in electrophysiology and cover the use of social media in these educational efforts.

Recent Findings Major international, national, and local meetings and electrophysiology fellowship-specific educational sessions have transitioned rapidly to virtual and distanced learning, enhanced by social media. This has allowed for participation in educational activities by electrophysiologists on a wider, more global scale. Social media has also allowed rapid dissemination of new advances, techniques, and research findings in real time and to a global audience, but caution must be exercised as pitfalls also exist.

Summary The digital and social media transformation of cardiac electrophysiology education has arrived and revolutionized the way education is delivered and consumed. Continued hybrid in-person and virtual modalities will provide electrophysiologists the flexibility to choose the best option to suit their individual needs and preferences for continuing education.
\end{abstract}

\title{
Introduction
}

Online and social media (SoMe) tools have impacted almost every aspect of daily life-medical education is no exception. The COVID-19 pandemic has further catalyzed the use of digital and SoMe platforms to facilitate major medical conferences and to support delivery of educational content in the face of travel bans, restrictions on in-person gatherings, and social distancing mandates. Success stories about the use of SoMe to facilitate medical education activities in the COVID-19 era are well-acknowledged. However, broader use of online and social media platforms has also brought to light a new set of pitfalls and challenges, specific to these digital tools, which will need to be addressed as SoMe becomes a more permanent fixture in the landscape of medical educational post-COVID-19. In this paper, we review the use of online platforms and SoMe tools to facilitate medical education with a particular focus on cardiac electrophysiology (EP). We also review the unique set of limitations and challenges relevant to the use of SoMe for medical education and offer potential solutions.

\section{Major medical conferences: a growing multimedia experience}

Annual medical scientific sessions have seen a natural and increasing incorporation of digital technology to enhance the educational experience over the past decade. With the current ubiquitous use of smartphones by physicians 
for both clinical practice and medical education, medical conferencing has also experienced a mobile revolution [1]. The paper-based systems of pamphlets, booklets, and syllabi in bookbags familiar to all conference attendees have been replaced by the incorporation of electronic conferencing mobile applications (mApps), freely available to download by anyone owning a smartphone. These mApps have streamlined conferencing "chores," allowing attendees to quickly search for, bookmark, and synchronize educational sessions to electronic calendars with chiming alerts; navigate conference venues; and even claim educational credit wirelessly at the touch of a button. Mobile application-integrated audience response systems, chat boxes, and question and answer (Q\&A) functionality have provided attendees with an improved and more interactive conference experience. Attendees now have the ability to propose questions to lecturers and panelists without having to step up to the microphone-possibly encouraging participation from those who may have been reticent in the past. The inclusion of live-streamed cardiac procedures during scientific sessions has given attendees key insights into the thought processes of experts as they perform these procedures in real time, occasionally from thousands of miles away, while the case is moderated by a panel of leaders in the field. Many medical societies have also made live-streaming of scientific sessions available to registrants, expanding their content to a truly global audience, allowing attendance live from anywhere in the world where internet connectivity is available [2,3].

The ongoing COVID-19 global pandemic helped spur further digital transformation of medical scientific sessions. This became more urgent and necessary as national and international travel bans, lockdowns, and social distancing mandates took effect. On March 9, 2020, the American College of Cardiology (ACC) became one of the first major medical conferences to cancel their annual in-person scientific sessions "with regard to the health, safety and well-being of our members, staff, exhibitor, faculty, patients and other key stakeholders," quickly transitioning to a free, 100\% virtual platform that could be experienced live or on-demand [4]. The Heart Rhythm Society (HRS) followed suit, cancelling their in-person scientific sessions 2 months ahead of schedule in favor of an all-virtual platform [5]. For both conferences, late-breaking clinical trial presentations were pre-recorded, panel sessions were conducted over video conferencing, scientific posters were made available for viewing online, and Q\&A sessions were performed through webinars-all of which were actively promoted via SoMe channels. Most importantly, conference content was uploaded to society websites and interactive online educational platforms such as Heart Rhythm Society 365 (HRS365) and YouTube (Google LLC, San Francisco, CA), and made available for viewing on-demand at attendees' own discretion-all at once, or in small portions as their schedules allowed [6, 7]. Virtual transformation appears to have increased educational accessibility and reach exponentially. While in-person HRS 2019 scientific sessions had 8089 attendees, virtual HRS 2020 educational sessions have garnered nearly 23,000 views, with ePosters amassing another 94,221 views-a metric previously unable to be quantified in live scientific sessions. Over the past year, all major cardiovascular societies, including the American Heart Association (AHA), the 
American Society of Echocardiography (ASE), the European Heart Rhythm Association (EHRA), the European Society of Cardiology (ESC), the Society of Cardiac Angiography and Intervention (SCAI), and Transcatheter Therapeutics (TCT), cancelled their in-person conferences, moving to completely virtual and often free or reduced registration rate platforms for their 2020 and 2021 scientific sessions [8-13]. Most recently, as the pandemic has waned in the USA, the 2021 Western Atrial Fibrillation Symposium successfully held a hybrid in-person and virtual event, combining global live streaming and on-location panel interaction [14]. In addition, HRS has announced that their 2021 scientific sessions will be a hybrid of both inperson and virtual, to better cater to educational needs worldwide [15]. Notably, these conferences have had registrants from all over the globe including countries previously less represented in these meetings-a sign of possible acceptance and greater ease of attending a virtual scientific sessions format.

While nothing can replace human interaction and connections made with face-to-face networking, these successes likely mean that virtual meetings are not just a stop gap in the era of COVID-19, but that digital conferencing will be a continued legacy resulting from the pandemic. This digital transformation has wide-ranging implications, including increased accessibility of educational content to a wider, more global audience, decreased expenses to conference attendees and supporting institutions, and reduced environmental impact due to less conference-associated travel. Digital sessions as a part or the whole of major scientific sessions are likely here to stay.

\section{Fellowship didactics: birth of a global virtual fellowship}

The rapid pivot to a digital platform has not been limited to national and international academic conferences; digital transformation also occurred at a local level. Institutional and EP trainee educational conferences, including grand rounds, morbidity and mortality $(M \& M)$ conferences, and case conferences were deferred during the early weeks of COVID-19 restrictions. As the need to reintroduce these educational conferences became apparent, intrainstitutional conferences were digitally transformed using various available video-conferencing modalities, such as Microsoft Teams (Microsoft Corp., Redmond, WA), Webex (Cisco Systems, Milpitas, CA), or Zoom (Zoom Video Communications, San Jose, CA). While this was most facile for institutions with pre-existing video-conferencing infrastructure, many still were able to seamlessly transition to a fully virtual platform allowing continuation of appropriately socially distanced learning [16]. In addition, in this new world of virtual education, invited grand rounds and lectureships have continued and have likely become less burdensome, allowing virtual visiting professors the ability to lecture at different institutions without the need to travel. 
Birth of shared lectureships

EP fellowship education adapted rapidly to the limitations in group gatherings due to COVID-19. The most immediate changes in fellowship training related activities occurred in hospitals affiliated with Accreditation Council for Graduate Medical Education (ACGME) accredited programs. Non-urgent EP procedures were largely scaled back, leading to an extremely limited clinical case volume for EP fellows, despite the continuation of daily virtual patient care rounds and local EP fellowship core curriculum lectures [17•]. Teaching faculty struggled to fill the available time with additional sessions to support ongoing fellow education. In a ground-breaking initiative, academic training institutions began providing inter-institutional, national, and international shared EP fellowship educational lectures and conferences via video conferencing. This education was provided in real time, on a regular basis and was freely available to any EP fellow in-training as well as interested faculty members. This allowed for continuation of required fellowship education, as well as an opportunity for fellows to network with fellowship classmates from across the nation (with whom they would otherwise have minimal interaction), and importantly with faculty from other institutions. A particularly notable example is the shared EP fellow lecture series initiated by Northwestern University (Chicago, IL). This was publicized to all EP training programs via Twitter and the HRS EP Fellowship Program Directors Committee. Lectures were delivered by experts in the field, highly attended by a national and international EP fellow and faculty audience, were archived for later viewing on YouTube for those who were unable to attend the real-time sessions, and are considered to be an excellent supplement to fellowship core curriculums across the globe.

Adaptation of industry sponsored courses tailored for EP fellows

An important part of EP didactic training is through industry-sponsored courses that allow fellows to interact with world-renowned lecturers on topics similar to EP fellowship core curriculum, supplemented by discussion of complex cases and electrograms. One course with a long tradition is the Interpreting the Unknown Electrograms Course, commonly held 1 day prior to the HRS scientific sessions and typically attended by approximately 200 EP fellows. In 2020, the 33rd anniversary of this course was held live, but virtually through Webex, with participation of 4 faculty and over 1000 national and international EP fellows. To extend international reach, this live course was repeated with the same faculty for the Asia-Pacific time zone, garnering the attendance of an additional $400+$ fellows. The annual Learn From The Experts: Approach to the Unknown Tracings conference, commonly held during HRS scientific sessions, was also held virtually on Zoom and was attended by over 600 fellows. Recently, EP 301: Complex concepts for EP fellows and Early Career EPs was conducted on Adobe Connect (Adobe Inc., San Jose, CA) and allowed 
participants to simultaneously view the slides and live video of the presenter, live chat with faculty, generate Q\&A, and participate in polls. Furthermore, The Basic Concepts Course, another EP fellow educational symposium held annually for the past 20 years, leveraged remote device interrogation technology and Zoom video conferencing in an attempt to create a hands-on EP fellow educational experience similar to the in-person experience where fellows would normally be facing device programmers, practicing troubleshooting of challenging device interrogations.

During the COVID-19 pandemic, the advantages of peer-to-peer interaction and networking in live courses have been traded for a broader reach of virtual courses $[17 \bullet$, 18]. Overall, educational programs have evolved into shorter, more focused sessions covering a handful of topics, rather than longer sessions over the course of 1-2 days broadly covering all topics. Through the use of virtual technologies, and with institutional and industry sponsor collaboration, seamless local and international educational opportunities are possible, with a majority of sessions archived for later viewing. Virtual learning platforms that have been necessary during the COVID-19 pandemic will likely stay after restrictions are lifted-to engage national and international audiences, to decrease barriers to participation and to make use of the technology that was rapidly developed and embraced to accommodate COVID-19-era teaching.

\section{Social media: a tool to supplement electrophysiology education}

The utility of SoMe to support education extends well beyond fellowship training and can be equally important for electrophysiologists in clinical practice [19]. SoMe education offers a wide range of educational material to suit the learner's needs, from didactic lectures (e.g., podcasts) to interactive tutorials (e.g., webinars), and written information (e.g., chat rooms). The virtual nature of SoMe education allows for attendance by anyone, from anywhere, at any time, provided there is access to the internet, a computer, a tablet, or a mobile phone.

\section{Social media to facilitate EP education}

Several platforms are available to provide education oer SoMe (Table 1) [20]. Sharing interesting and challenging EP cases, intracardiac tracings, and imaging over interactive SoMe platforms, such as Facebook (Facebook, Inc., Menlo Park, CA), Twitter, or WhatsApp (WhatsApp LLC, Mountain View, CA), provides rapid and dynamic feedback from a global community of peers and experts on SoMe. YouTube videos have been used to provide medical education in the form of lectures, grand rounds, and animated or "real-life" prerecorded procedural videos. In addition, planned or more formal discussions 


\section{Table 1 Social media formats}

\begin{tabular}{|c|c|}
\hline Site & Description \\
\hline Doximity $^{a}$ & $\begin{array}{l}\text { Online networking, restricted to medical professionals; posting of educational publications, medical } \\
\text { news, and case discussions; provides networking between members via messaging service }\end{array}$ \\
\hline Facebook $^{b}$ & $\begin{array}{l}\text { Online social networking; individuals can create public or private profiles, and network with others by } \\
\text { sharing posts, images, and videos. Major medical societies and journals create pages to post scientific } \\
\text { or society news and post key findings from manuscripts. Groups, both public and private, exist for case- } \\
\text { based formal and informal presentations and discussions (e.g., Heart Rhythm Café, 'Shock Absorbers' ICD } \\
\text { support group.) }\end{array}$ \\
\hline HRS communities & $\begin{array}{l}\text { Online forum restricted to HRS members who can participate in discussions relevant to issues brought } \\
\text { up by the EP community; multiple communities exist (e.g., General Membership, Allied Professionals, } \\
\text { Early Career, Research, etc.) and are open to all members; closed groups for HRS committee work also } \\
\text { exist }\end{array}$ \\
\hline Heart rhythm TV & $\begin{array}{l}\text { Flagship HRS YouTube channel with Breaking News reports, summaries of scientific presentations, and } \\
\text { other educational content specific to the cardiac electrophysiology community }\end{array}$ \\
\hline LinkedIn ${ }^{c}$ & $\begin{array}{l}\text { Online professional networking; individuals can create profiles, post of curriculum vitae, find employment, } \\
\text { and network electronically via direct messaging. Members can also write posts/articles and share videos }\end{array}$ \\
\hline \multirow[t]{3}{*}{ Twitter $^{d}$} & $\begin{array}{l}\text { Twitter: online social networking in the form of microblogging limited to } 280 \text { characters per "tweet." } \\
\text { Profiles can be public or private and exist for individuals, societies, journals, and institutions. Often, } \\
\text { de-identified discussions of cases occur with de-identified images and videos, usually containing } \\
\text { learning points or tips on achieving successful outcomes }\end{array}$ \\
\hline & $\begin{array}{l}\text { Twitter Chat: scheduled interactive sessions with planned topics of discussion and panelists; allows } \\
\text { for discussion on a public platform and participation of a wide virtual audience }\end{array}$ \\
\hline & $\begin{array}{l}\text { Twitter Journal Club: formal review and discussion of journal articles, akin to in-person journal clubs, } \\
\text { often with participation of journal authors; some are general EP, others disease or device specific }\end{array}$ \\
\hline Weibo ${ }^{\mathrm{e}}$ & $\begin{array}{l}\text { Online social networking in the form of microblogging; similar in format to Twitter, but used exclusively } \\
\text { by Chinese-language speakers }\end{array}$ \\
\hline WhatsApp ${ }^{f}$ & $\begin{array}{l}\text { Online messaging and voice-over-IP; users can share text messages, images, videos, or create voice } \\
\text { messages. Chat groups exist for heart rhythm professionals with a common interest }\end{array}$ \\
\hline YouTube $^{g}$ & $\begin{array}{l}\text { Online social media and video-sharing channel; educational video can be posted by individuals, societies, and } \\
\text { institutions. Examples specific to cardiac electrophysiology include BiVTips, CardioVisual, Heart Rhythm TV, } \\
\text { and Expert Perspectives }\end{array}$ \\
\hline
\end{tabular}

Corp. Corporation, EP electrophysiology, HRS Heart Rhythm Society, ICD implantable cardioverter-defibrillator, Inc. incorporated, LLC Limited liability company

${ }^{a}$ Doximity, San Francisco, CA

${ }^{b}$ Facebook, Inc., Menlo Park, CA

'LinkedIn Corp., Sunnyvale, CA

${ }^{\mathrm{d}}$ Twitter, Inc., San Francisco, CA

eWeibo, Sina Corp., China

${ }^{f}$ WhatsApp LLC, Mountain View, CA

${ }^{g}$ Google LLC, San Francisco, CA

over SoMe have the potential to inform a far greater number of people than traditional lectures that may be limited to those with the resources and time to attend. The HRS \#LifestyleAndAFib Twitter Chat in October 2019 had nearly 400 participants and generated 17.8 million impressions (a measure of 
potential reach). Twitter chats discussing other current EP issues (e.g., Cardiac EP in the COVID-19 Era) have also attracted significant participation in SoMe from EP community members, generating provocative discussions and providing new insights into patient care in these uncertain times. Twitter journal clubs have also been an important source of discussion and dissemination of newly published science, and often involve the live participation of manuscript authors-a distinct benefit over traditional journal clubs. International participation, another distinct advantage of online journal clubs, may help enhance knowledge translation and professional development. More recently, continuing medical education (CME) or Maintenance of Certification (MOC) points can be gained by participating in online learning, improving access to satisfy institutional or regulatory board requirements [21••]. Several small studies have also shown that published manuscripts that are publicized on Twitter receive more citations than comparable manuscripts that are not; whether or not this changes an author's h-index or a journal's impact factor remains to be seen $[22,23 \bullet \bullet, 24]$.

Social media use and scientific sessions

Initially, many medical societies resisted or banned the use of SoMe during medical meetings, citing worries of violating scientific embargoes and fears of driving down in-person attendance [25]. A recent analysis of 3 major cardiovascular conferences showed that Twitter (Twitter, Inc., San Francisco, CA) use tripled between the years 2014 and 2016, with 75\% of activity during conferences consisting of educational tweets, reaching a global virtual audience of nearly 250 million. Importantly, in-person attendance remained stable [26••]. Other studies have shown that SoMe use during scientific sessions is a powerful tool to amplify research findings, increase reach of educational content, and an important mechanism to keep conference attendees connected by facilitating professional networking and generating robust scientific discussions $[27-29 \bullet, 30]$. Understanding this added value, major medical societies have since embraced the use of SoMe, creating official conference hashtags and running live SoMe feeds during sessions. The first virtual HRS 2020 late breaking clinical trial session had 7288 views on HRS365 and YouTube, with multiple discussions occurring organically on Twitter, reaching a much larger audience than a singular in-person scientific session. In addition, cardiovascular societies including the ACC, AHA and ESC have appointed official conference "Twitter ambassadors." In this model, clinician members are officially charged with disseminating scientific session content on Twitter in real time to help facilitate discussions in this virtual space and engage a larger, more global virtual audience [31].

While the lack of in-person attendance limits networking and personal interaction, the ease and convenience of SoMe and its ability to disseminate content nearly instantaneously and in real time have made scientific sessions more accessible to audiences across continents. As is already occurring, a combination of in-person meetings with virtual components will 
provide electrophysiologists the ability to choose the best option to suit their individual needs and preferences for continuing education.

\section{Digital/virtual education: perils, pitfalls, and limitations}

\section{Access to digital resources}

While SoMe efforts have gained traction as an effort to engage a wider virtual audience, it must be noted that some jurisdictions place restrictions on SoMe platforms. For example, China blocks most SoMe applications such as Facebook and Twitter, while some others, like WhatsApp, have only partial functionality. There are geographical variations in the popularity of various platforms which should be taken into account; offering educational content across a variety of platforms is likely to gain the greatest reach. Country-specific platforms (e.g., Weibo, Sina Corp., China) may be also utilized to decrease these barriers.

\section{Psychological impact of online interaction}

Smartphones have become ubiquitous; tablets and laptops have become more portable. SoMe platforms like Facebook and Twitter are no longer explored from time to time on stationary computers, but minute-to-minute on mobile personal devices. Constant accessibility has significantly increased time spent online [32]. This has led to associated physical and psychological ailments, including De Quervain's syndrome, gaming, and SoMe disorder, as well as SoMe-induced depression and anxiety [33-35]. Online harassment, bullying, or "cancel culture" are not uncommon, and the medical community, including the EP community, is not immune. In addition, cyberattacks by "zoom bombers" (unwanted interlopers) projecting abusive, hateful, or offensive audio and visual content have caused disruption and cancellation of virtual conferences [36].

It is unknown how the above-mentioned aspects may impact heart rhythm specialists who are active online. Organizations like HRS have a responsibility to remind their members that time away from work and education is important for a balanced lifestyle. Tendency for online and SoMe applications to interrupt family time or relaxation should be recognized, and awareness of these issues should be promoted. Members should be able to tailor their virtual and SoMe educational platforms to suit their personal circumstances, time zones, work week variations, and interruptionfree times. The danger of always being "on" and the desire to educate must be balanced with personal time to prevent additional triggers for burnout. 
Currently, there is no consensus regarding property rights of original educational or scientific content shared in the digital space. There has been debate about whether sharing preliminary study results and techniques on digital platforms violates journal publication policies and whether this places future publication of related manuscripts in jeopardy. This question has also surfaced in the context of sharing scientific posters and presentation slides from conferences on SoMe. The discussion on possible violations led to a change in policy of some of the most important scientific meetings [26••, 37]. Apart from situations clearly violating conference regulations, it is unclear if such content sharing is acceptable [38]. Posting educational content without referencing the original author remains a problem in some medically oriented online groups.

SoMe has become a platform where EP clinicians have gathered to share new techniques and treatments, ask for or offer advice on difficult or complex cases, and amplify and discuss findings of research studies. The rapid rebirth and uptake of physiologic pacing has been credited to the sharing of His bundle pacing successes and failures by the EP community on Twitter. Sales of the Medtronic (Minneapolis, MN) 3830 pacing lead grew exponentially overtime, correlating with the growth in number of users and impressions from the His bundle pacing hashtag \#dontdisthehis [39••]. Cardioneuroablation for treatment of cardioinhibitory vasovagal syncope and functional AV block has also gained significant interest, partially through dissemination of technique and outcomes on SoMe, despite minimal presence in the current syncope guidelines [40]. However, these "successes" of SoMe in EP must also be visualized through a critical lens. Although free online sharing of physicians' case presentations, commentary, and insights can be educational, none of this is subject to peer review. Most clinical cases shared on Twitter have no follow-up [41]. Dissemination and potential widespread adoption of a procedure, medication, or treatment plan without peer review or the demonstration of safety and efficacy through either large observational studies, or better yet, randomized controlled trials, could be risky, and many approaches could be considered "off label." Can we as a community allow practitioners to "self-referee"? If so, determining who is responsible for vetting the message of medical content on SoMe, at what time and in what form requires much greater thought [42].

Beyond online presentation of individual cases or techniques, similar challenges also hold true for broader dissemination of scientific content on online. Scientific studies, such as the wearable cardioverter-defibrillator after myocardial infarction (VEST) and the Catheter Ablation vs. Antiarrhythmic 
Drug Therapy for Atrial Fibrillation (CABANA) trials, have undergone public discussions immediately as they are released as late breaking clinical trials, serving as a form of "post-publication peer review" with critical analysis akin to a free-flowing public journal club by EPs on SoMe [43]. Sharing the results of a major clinical trial via SoMe can be extremely powerful, gaining rapid traction through amplification from re-tweeting and further commentary. This could be extremely important, especially if a drug or treatment is deemed to be harmful, allowing many clinicians to rapidly stop such practice. However, this practice may also have negative effects if the results are misinterpreted, miscommunicated, objectively false, or relayed with malcontent from disagreement with the results. The same caution should be exercised when observational and retrospective data are shared as if they were the results of a well-done, blinded, placebo-controlled, randomized clinical trial. This too can spread rapidly through SoMe and be practice changing, but without sufficient scientific basis. SoMe should not be used in isolation, but rather, as a tool to complement education, leading the learner to continue to seek out multiple, vetted sources of information $[42,44]$.

A major attraction of SoMe is the ability to rapidly post, review, and encourage fast-moving commentary from a richly diverse and intercontinental audience. This is a tremendous boon to EP and is a critical part of future advances. The concern about author (or person posting) conflict of interest (COI) has been raised $[45,46]$. In some cases, the posting person may have relevant COI related to a SoMe post via prior work, compensation, or research funding from a company. This is an understandable concern that raises questions as to whether or not a COI statement should, or even could, be made in these posts. Certainly, a clear COI should be made upfront by the author as a means of professionalism and character; however, mechanisms for regulating and managing COI in SoMe remain nascent.

Implementation of COI declarations on SoMe platforms poses practical challenges. SoMe author bios are character-limited and would not allow for insertion of multiple COI statements. Potentially, software could be engineered for a "pop-up" COI statements with each post [46]. Another potential solution would be to add \#C (noting COI) or \#NC (no COI), which the author would almost certainly need to clarify in a further subpost. However, requirements to provide COI clarification each time may lead to fewer posts if "extra work" is required. In addition, the reader is unlikely to review all of these COIs. Opportunities for managing COI in SoMe should draw upon lessons learned from other medical media such as print journals and television.

In general, SoMe is less prone to direct forms of COI compared to other medical media. In contrast to major journals where there are sometimes cover pages, inserts, and several full-page ads of devices, ablation tools, or medications, such advertising needs are less germane for individual tweets 
or posts. Other issues related to COI are more specifically relevant to SoMe. Could the "following" or "friending" of industry sites or representatives be construed as a type of COI, or should a different subset of additional rules and regulations be applied? Similar questions can be raised about "liking" or re-tweeting posts about new ablations tools, mapping systems, or devices and whether this represents a form of tacit endorsement or approval. In many cases, a person posting on SoMe may not have any financial ties to a specific product or company. The ramifications of COI disclosure for these types of SoMe interactions represent a gray area in what has traditionally been covered by COI rules. Depending on the number of followers and influence, some forms of SoMe activity may have much broader impact and relevance than activities which have traditionally been governed by COI, such as speaking at an industry-supported dinner [44]. Developing approaches for managing $\mathrm{COI}$ on SoMe is likely to pose significant challenges and will require creative and out-of-the-box thinking.

\section{Conclusions}

The COVID-19 pandemic has helped to propel the digital and SoMe transformation of EP education, further revolutionizing the way education is delivered and consumed. While there have been a multitude of benefits to this transformation, clear limitations also exist, and further work is needed to explore both positive and negative impacts as use continues to increase in clinical EP. Overall, continued hybrid in-person and virtual modalities will provide electrophysiologists the flexibility to choose the best option to suit their individual needs and preferences for continuing education even in the post-pandemic world.

\section{Compliance with Ethical Standards}

Conflict of interest

Christopher V. DeSimone, Susan P. Etheridge, Piotr Futyma, Rakesh Gopinathannair, Rajesh Kabra, Sandeep A. Saha, Faisal M. Merchant declares that they have no conflict of interest. Tina Baykaner reports grants from the National Institutes of Health, lecturing fees from Biotronik, and consulting fees PaceMate. Janet K. Han reports lecturing fees from Abbott, Boston Scientific and Medtronic. Rajesh Kabra reports grants from Abbott, Biosense Webster and Medtronic. Martin K. Stiles reports lecturing and consulting fees from Boehringer Ingelheim, consulting fees from Ceryx Medical and lecturing fees from Medtronic.

\section{Research Involving Human and Animal Participants}

This article does not contain any studies with human or animal subjects performed by any of the authors. 


\section{References and Recommended Reading}

Papers of particular interest, published recently, have been highlighted as:

- Of importance

•• Of major importance

1. Baumgart DC. Smartphones in clinical practice, medical education, and research. Arch Intern Med. 2011;171:1294-6.

2. American Heart Association and American Stroke Association OnDemand. Earn cardiology CME with AHA and ASA on demand [Internet]. [cited 2021 Jun 21]. Available from: https://aha.ondemand.org/aha/

3. iScience [Internet]. Am Coll Cardiol [cited 2021 Jun 21]. Available from: https://www.acc.org/education-andmeetings/products-and-resources/http $\% 3 \mathrm{a} \% 2 \mathrm{f} \% 2 \mathrm{fwww}$. acc.org\% $\%$ feducation-and-meetings $\% 2$ fproducts-andresources\%2fiscience

4. Virtual ACC.20/WCC: The Show Must Go On [Internet]. Am. Coll. Cardiol. [cited 2021 Jun 21]. Available from: https://www.acc.org/Membership/ Sections-and-Councils/Cardiovascular-Team-Section/ Section-Updates/2020/05/06/12/42/http\%3a\%2f\% 2 fwww.acc.org\%2fMembership\%2fSections-andCouncils\%2fCardiovascular-Team-Section $\% 2$ fSectionUpdates $\% 2$ f2020\%2f05\%2f06\%2f12\%2f $42 \%$ 2 firtual-ACC20-WCC-The-Show-Must-Go-On

5. 2020 Heart Rhythm Scientific Sessions|Heart Rhythm Society [Internet]. [cited 2021 Jun 21]. Available from: https://www.hrsonline.org/education/live-meetings/ 2020HeartRhythmScientificSessions

6. ACC.20/WCC Meeting Coverage [Internet]. Am Coll Cardiol [cited 2021 Jun 21]. Available from: https://www. acc.org/http\%3a\%2f\%2fwww.acc.org\%2fACC2020\%3f_ ga\%3d2.110040197.1874734723.1597781501976128704.1590106574

7. HRS 2020 Science|Heart Rhythm Society [Internet]. [cited 2021 Jun 21]. Available from: https://www. hrsonline.org/HRS2020Science

8. Scientific Sessions 2020 Newsroom [Internet]. Am Heart Assoc [cited 2021 Jun 21]. Available from: https://newsroom.heart.org/events/scientific-sessions2020-newsroom

9. ASE 2020 Virtual experience features top research from around the world [Internet]. [cited 2021 Jun 21]. Available from: https://www.asecho.org/ase-2020-virtualexperience-features-top-research-from-around-the-world/

10. Burri H, Zeppenfeld K, Heidbuchel H. EHRA 2020 during COVID-19 days. Eur Heart J. 2020;41:2828-9.

11. ESC Congresses \& Events [Internet]. [cited 2021 Jun 21]. Available from: https://www.escardio.org/Congresses- \&Events, https://www.escardio.org/Congresses-\&-Events

12. SCAI 2020 Scientific Sessions: Virtual Conference [Internet]. [cited 2021 Jun 21]. Available from: https:// virtual2020.scai.org/
13. TCT 2020 Is Now a Virtual Event: TCT Connect - CRF [Internet]. [cited 2021 Jun 21]. Available from: https:// www.crf.org/crf/news-and-events/news/news/3628-tct2020-is-now-a-virtual-event-tct-connect

14. Western Atrial Fibrillation Symposium [Internet]. [cited 2021 Jun 21]. Available from: https://westernaf. $\mathrm{com} /$

15. Heart Rhythm 2021|Heart Rhythm Society [Internet]. [cited 2021 Jun 21]. Available from: https://www. hrsonline.org/heart-rhythm-2021

16. Almarzooq ZI, Mathew L, Ajar K. Virtual learning during the COVID-19 pandemic. J Am Coll Cardiol. 2020;75:2635-8.

17. Shapiro H, Reza N. Cardiovascular medical education during the coronavirus disease 2019 pandemic: challenges, adaptations, and considerations for the future. US Cardiol Rev. 2021;15:e05.

This manuscript gives a detailed summary of the impact of the COVID-19 pandemic on cardiology trainees and includes an overview of transition to virtual education.

18. Kotta PA, Corso B, Brailovsky Y, Oliveros E. The impact of 2020 and the future of cardiology training. JACC Case Rep. 2021;3:697-700.

19. Davis WM, Ho K, Last J. Advancing social media in medical education. CMAJ. 2015;187:549-50.

20. Dol J, Tutelman PR, Chambers CT, Barwick M, Drake EK, Parker JA, et al. Health researchers' use of social media: scoping review. J Med Internet Res. 2019;21:e13687.

21.• Thamman R, Desai T, Wiener DH, Swaminathan M. \#ASEchoJC Twitter Journal Club to CME: a paradigm shift in cardiology education. J Am SocEchocardiogr. 2020;33:A29-35.

This study characterizes the use of Twitter for online journal club by the American Society of Echocardiography and describes and how they were the first cardiovascular society to use this as an avenue for continuing medical education credits. 22. Luc JGY, Archer MA, Arora RC, Bender EM, Blitz A, Cooke DT, et al. Social media improves cardiothoracic surgery literature dissemination: results of a randomized trial. Ann Thorac Surg. 2020;109:589-95.

23.• Ladeiras-Lopes R, Clarke S, Vidal-Perez R, Alexander $\mathrm{M}$, Lüscher TF. Twitter promotion predicts citation rates of cardiovascular articles: a preliminary analysis from the ESC Journals Randomized Study. Eur Heart J. 2020;41:3222-5.

This preliminary analysis showed that randomization to a strategy of SoMe promotion for cardiovascular medicine 
manuscripts was associated with an increase in both online impact and number of citations, compared to manuscripts that were not promoted on SoMe. The final analysis will be completed March 2021.

24 Klar S, Krupnikov Y, Ryan JB, Searles K, Shmargad Y. Using social media to promote academic research: Identifying the benefits of twitter for sharing academic work. PLOS ONE. 2020;15:e0229446.

25. Neporent, L. A perfect Twitter storm: why is the ADA so anti-social media? [Internet]. Medscape. [cited 2021 Jun 21]. Available from: http://www.medscape.com/ viewarticle/881418

26.• Tanoue MT, Chatterjee D, Nguyen HL, Sekimura T, West BH, Elashoff D, et al. Tweeting the meeting. Circ Cardiovasc Qual Outcomes. 2018;11: e005018.

This is the 1st study to characterize the increased use of Twitter in 3 major cardiovascular society scientific sessions over time, with the majority of over 80,000 tweets being educational in nature. In-person attendance at these conferences did not significantly decrease despite concerns of sharing of conference content on SoMe.

27. Mishori R, Levy B, Donvan B. Twitter use at a family medicine conference: analyzing \#STFM13. Fam Med. 2014;46:608-14.

28. Cawcutt KA, Erdahl LM, Englander MJ, Radford DM, Oxentenko AS, Girgis L, et al. Use of a coordinated social media strategy to improve dissemination of research and collect solutions related to workforce gender equity. J Womens Health. 2002;2019(28):849-62.

29.• Chandra NV, Hsiao R, Shapiro H, Snow S, Truong K, Beach S, et al. Women in cardiology Twitter network: an analysis of a global professional virtual community from 2016 to 2019. J Am Heart Assoc; 2021;10: e019321.

This is the 1st study to characterize the use of Twitter as new way to facilitate networking for women cardiology professionals. Looking at $>48,000$ tweets, Twitter was found to help networking via community building, professional development and advocacy.

30. Yeh RW. Academic cardiology and social media: navigating the wisdom and madness of the crowd. Circ Cardiovasc Qual Outcomes. 2018;11:e004736.

31. Linz D, Garcia R, Guerra F, Kommata V, Bollmann A, Duncker D. Twitter for professional use in electrophysiology: practical guide for \#EPeeps. EP Eur [Internet]. 2021 [cited 2021 Jun 21]; Available from: https://doi. org/10.1093/europace/euab048

32. Daily social media usage worldwide [Internet]. Statista [cited 2021 Jun 21]. Available from: https://www. statista.com/statistics/433871/daily-social-media-usageworldwide/

33. Ali M, Asim M, Danish SH, Ahmad F, Iqbal A, Hasan SD. Frequency of De Quervain's tenosynovitis and its association with SMS texting. Muscles Ligaments Tendons J. 2014;4:74-8.

34. Shensa A, Sidani JE, Dew MA, Escobar-Viera CG, Primack BA. Social media use and depression and anxiety symptoms: a cluster analysis. Am J Health Behav. 2018;42:116-28.

35. van den Eijnden RJJM, Lemmens JS, Valkenburg PM. The social media disorder scale. Comput Hum Behav. 2016;61:478-87.

36. Read B. 'Zoombombing' is a horrifying new trend [Internet]. The cut 2020 [cited 2021 Jun 21]. Available from: https://www.thecut.com/2020/04/what-iszoombombing.html

37. Ghose A, Warren HR, Raison NTJ, Dasgupta P. The controversy of social media at conferences. BJU Int. 2018;121:823-4.

38 Mackenzie DG, Hudson S, Gulati M. Who influences tweeting at international cardiology conferences? Eur Heart J. 2020;41:2423-7.

39.• Beer D, Dandamudi G, Mandrola JM, Friedman PA, Vijayaraman P. His-bundle pacing: impact of social media. EurEur Pacing Arrhythm Card Electrophysiol J Work Groups Card Pacing Arrhythm Card Cell Electrophysiol Eur Soc Cardiol. 2019;21: 1445-50.

This study shows the impact of SoMe on the adoption of new techniques. Specifically, they show that sales of the Medtronic 3830 His-bundle pacing lead correlated with number of tweets with the hashtag \#dontdisthehis, implying that sharing this technique on Twitter impacted the spread and integration of the HBP technique worldwide.

40. Shen W-K, Sheldon RS, Benditt DG, Cohen MI, Forman DE, Goldberger ZD, et al. ACC/AHA/HRS guideline for the evaluation and management of patients with syncope: executive summary: a report of the American College of Cardiology/American Heart Association task force on clinical practice guidelines and the Heart Rhythm Society. Circulation. 2017;136:e25-59.

41. Cifu AS, Vandross AL, Prasad V. Case reports in the age of twitter. Am J Med. 2019;132:e725-6.

42. Hill JA, Agewall S, Baranchuk A, Booz GW, Borer JS, Camici PG, et al. Medical misinformation. Circulation. 2019;139:571-2.

43. Mandrola J, Futyma P. The role of social media in cardiology. Trends Cardiovasc Med. 2020;30:32-5.

44 Zenger B, Swink JM, Turner JL, Bunch TJ, Ryan JJ, Shah RU, et al. Social media influence does not reflect scholarly or clinical activity in real life. Circ Arrhythm Electrophysiol. 2020;13:e008847.

45. Kaestner V, Brown A, Tao D, Prasad V. Conflicts of interest in Twitter. Lancet Haematol. 2017;4:e408-9.

46. McCarthy CP, DeCamp M, McEvoy JW. Social media and physician conflict of interest. Am J Med Elsevier. 2018;131:859-60.

\section{Publisher's Note}

Springer Nature remains neutral with regard to jurisdictional claims in published maps and institutional affiliations. 